\title{
Treino Locomotor com Suporte de Peso Corporal na Lesão Medular Incompleta
}

\author{
Locomotor Training with Body Weight-Support in Incomplete Spinal Cord Injury
}

\section{Renata Teles Vieira', Rafaela Machado de Gusmão Oliveira², Camila Alves Nogueira Barros', Leonardo Caixeta ${ }^{3}$}

\begin{abstract}
RESUMO
Objetivo. O objetivo deste trabalho foi realizar uma revisão de literatura sobre o uso do treino locomotor em pacientes portadores de lesão medular incompleta, a fim de verificar os seus efeitos para a marcha destes pacientes. Método. Foi realizada uma busca utilizando os bancos de dados medline, scielo e bvs a partir dos descritores: body weight-support treadmill training (suporte parcial de peso com treinamento em esteira), locomotor training (treino locomotor), spinal cord injury (lesão medular), gait (marcha). Todos os artigos coletados nos últimos 18 anos foram analisados. Discussáo. A lesão medular é uma grave síndrome neurológica que causa diversos comprometimentos, inclusive da marcha. Para aperfeiçoar este processo, deu-se início à prática de reabilitação na esteira com suporte de peso corporal. A ampla utilização desta técnica de reabilitação deve-se a maior facilidade para o treino da marcha, a satisfação dos pacientes durante o tratamento e, principalmente, aos bons resultados gerados. Conclusáo. Um número significante de estudos mostrou que o treino de marcha com suporte de peso corporal é um meio seguro e confiável, e que surgiu para inovar a reabilitaçáo funcional da marcha. Não há evidência científica para afirmar que o treino locomotor com suporte de peso seja um método superior a outras terapias.
\end{abstract}

Unitermos. Marcha, Atividade Motora, Lesôes da Medula Espinhal, Reabilitação.

Citaçáo. Vieira RT, Oliveira RMG, Barros CAN, Caixeta L. Treino Locomotor com Suporte de Peso Corporal na Lesão Medular Incompleta.

\begin{abstract}
Objective. The objective was to perform a literature review on the use of locomotor training in patients with incomplete spinal cord injury in order to verify their effects for the running of these patients. Method. We performed the search portal's medline, scielo and bvs from the key words: body weight-supported treadmill training - BWSTT, supported treadmill ambulation training - STAT, support of body weight - SPC, spinal cord injury. Articles that could be collected in full in the last 18 years were analyzed. Discussion. Spinal cord injury is a severe neurological syndrome that causes several serious compromises including the gait. To improve this process, it has begun the practice of rehabilitation in connection with support of body weight. The wide use of this technique for rehabilitation due to ease of travel for training, the satisfaction of patients during treatment and, especially, the results generated. Conclusion. Studies show that he gait training with body weight support is a safe and reliable means that appeared to innovate and functional rehabilitation of gait. There is no scientific evidence to assert that the BWSTT is a superior method to other therapies.
\end{abstract}

Keywords. Gait, Motor Activity, Spinal Cord Injuries, Rehabilitation.

Citation. Vieira RT, Oliveira RMG, Barros CAN, Caixeta L. Locomotor Training with Body Weight-Support in Incomplete Spinal Cord Injury.
Trabalho realizado na Universidade Federal de Goiás, Goiânia-GO, Brasil. 1. Fisioterapeuta, Doutoranda em Ciências da Saúde pela Universidade Federal de Goiás, Goiânia-GO, Brasil.

2. Fisioterapeuta, Especialista em Fisioterapia Neurológica, Centro de Estudos Avançados e Formação Integrada - Goiânia-GO, Brasil.

3. Médico Neuro-Psiquiatra, Doutor em Neurologia pela USP, Professor adjunto da Universidade Federal de Goiás, Goiânia-GO, Brasil.
Endereço para correspondência: Renata T Vieira

1a Avenida, s/n - Setor Leste Universitário CEP 74605-020, Goiânia-GO, Brasil.

E-mail: renatavip5@hotmail.com 


\section{INTRODUÇÃO}

As lesões medulares (LM) são cada vez mais frequentes devido principalmente ao aumento da violência urbana. Dentre as principais causas, encontramos o acidente de trânsito e a agressão por arma de fogo. Os pacientes acometidos, em sua maioria, são jovens, do sexo masculino, solteiros e residentes em áreas urbanas ${ }^{1}$.

As LM geram uma incapacidade de alto custo para o governo e acarreta importantes alteraçôes no estilo de vida do paciente. Tais lesóes causam perda parcial ou total da motricidade e sensibilidade, além de comprometimento vasomotor, intestinal, vesical e sexual ${ }^{2}$. Além de sua gravidade e irreversibilidade, as LM exigem um programa de reabilitaçâo longo e oneroso, que na maioria das vezes não leva à cura, mas à adaptação do indivíduo à sua nova condição. Esse processo de reabilitação, no entanto, vai para além da prevenção dos danos causados pela lesão e objetiva principalmente melhora da qualidade de vida através da independência funcional, melhora da autoestima e inclusão social desses pacientes ${ }^{1}$.

As lesões medulares são classificadas segundo dois critérios: nível neurológico da lesão e lesão completa ou incompleta. O nível neurológico da lesão é determinado pelo mais caudal segmento sensitivo e motor preservado bilateralmente. Porém, o funcionamento motor pode estar comprometido em nível diferente do sensorial e as perdas podem ser assimétricas ${ }^{3}$.

A recuperação da marcha é uma tarefa difícil e dispendiosa, os pacientes muitas vezes são incapazes de produzir a força muscular necessária para manter a postura e caminhar ${ }^{4}$. Para estes casos, é necessário prover um suporte para proteger estes sujeitos de quedas por meio dos sistemas convencionais de ajuda para auxílio na marcha como barras paralelas, muletas e bengalas, a quantidade de peso aliviada não é constante e nem facilmente quantificável. Outro fato é que estes dispositivos dependem da força, controle de tronco e coordenação dos pacientes. Além disso, o consumo energético do paciente com a utilização destes auxílios para a marcha é maior. Assim, com estes dispositivos, muitas vezes, não se consegue gerar as condiçôes para um treino de marcha seguro e eficaz 5 .

Restaurar a deambulação quando o paciente apresenta prognóstico para a marcha, requer variadas técnicas e geralmente exige assistência considerável do terapeuta para segurar o peso do paciente e aumentar seu equilíbrio. No treino de marcha convencional, muitas vezes, o resultado não satisfaz o paciente, com padróes assimétricos de movimento e principalmente, com dificuldade de percorrer maiores distâncias ${ }^{6,7}$. Para aperfeiçoar este processo, deu-se início à prática de reabilitação na esteira e mais tarde, o treino de marcha na esteira com suporte de peso corporal (TMSPC) ${ }^{8}$.

Treino de marcha na esteira com suporte de peso corporal (body weight-supported treadmill training - BWSTT, supported treadmill ambulation training STAT ou Laufband therapy) é um sistema de suspensão, o qual reduz a força resultante entre a força gravitacional e a força de suspensão, diminuindo a carga sobre o aparelho musculoesquelético durante o treino de marcha em esteira. A suspensão segura parcialmente o peso do paciente e com isso a marcha é facilitada ${ }^{9,10}$. A facilidade da marcha ocorre também devido ao maior controle de tronco $^{11,12}$, ao auxílio da marcha pela esteira ${ }^{13}$ e ao auxílio do terapeuta que pode atuar nas características da marcha que foram diagnosticadas como deficitárias ${ }^{14}$.

A base teórica para o surgimento do TMSPC advém de estudos com gatos com lesão medular que passaram por este treino de marcha ${ }^{15}$. A partir destes estudos, foi constatado que existia no sistema nervoso dos animais um gerador de padrão central que era responsável por gerar o padrão cíclico da marcha para estes animais, mesmo com pouco treino após a lesão medular ${ }^{16-18}$.

Depois do sucesso da reabilitação com os animais, por volta dos anos 80 , surgiram os primeiros estudos em seres humanos a partir dos trabalhos de Lois Finch e $\mathrm{Hu}$ gues Barbeau ${ }^{12,19-23}$.

Historicamente o TMSPC está vinculado a acometimentos neurológicos, sendo utilizado primeiro para pacientes com acidente vascular cerebral ${ }^{11,24}$ e lesados medulares ${ }^{20,23}$, depois iniciou o tratamento de diversas doenças neurológicas e ortopédicas. A ampla utilização desta técnica de reabilitação deve-se a maior facilidade para o treino da marcha, a satisfação dos pacientes durante o tratamento e principalmente, aos bons resultados $\operatorname{gerados}^{25}$.

Com esta nova opçấo de atividade para a recuperação da marcha, este estudo teve por objetivo realizar uma revisão de literatura sobre o uso do treino locomotor em 
portadores de lesão medular incompleta a fim de verificar o seu efeito nestes pacientes.

\section{MÉTODO}

Para este estudo de revisão foi realizada uma busca utilizando os bancos de dados Medline, Scielo, BVS, além de revistas, sites e congressos brasileiros da área de saúde. Foram selecionados artigos a partir dos descritores: body weight-support treadmill training (suporte parcial de peso com treinamento em esteira), locomotor training (treino locomotor), spinal cord injury (lesão medular), gait (marcha). Os artigos datados de 1991-2009 foram selecionados. Os critérios para inclusão dos artigos incluíam: artigos originais escritos em português, inglês e/ou espanhol, mostrar intervenção do TMSPC em pacientes com lesão medular incompleta por qualquer etiologia.

\section{RESULTADOS}

Foram encontrados 47 artigos. Foram excluídos os artigos que apresentavam pacientes com classificações ASIA A e/ou $\mathrm{B}^{26}$, artigos comparando o suporte de peso corporal manual com o robótico e artigos associando a utilização de drogas ao treino de marcha na esteira com SPC também foram excluídos. Restando após todos os critérios da exclusão: 21 artigos. Todos os artigos encontrados estão listado no Quadro 1, com seus respectivos métodos e resultados.

\section{DISCUSSÃO}

Dezesseis dos vinte e um artigos encontra$\operatorname{dos}^{20,22,27-35,39,39,41-43}$ demonstraram que o tratamento com TMSPC em LM incompleta (ASIA C e D) apresenta algum efeito positivo sobre os parâmetros espaciais e temporais da marcha, como simetria, consumo de $\mathrm{O}_{2}$ (menor dispêndio de energia), cadência, coordenação motora, balanço dos braços, funcionalidade motora, comprimento do passo, distância percorrida e velocidade da marcha.

Os artigos remanescentes apontaram outros efeitos do TMSPC, além de características da marcha. Melhora na regulação sanguínea da glicose ${ }^{37}$, ausência ou pouca melhora na qualidade de vida ${ }^{40,42}$, alteração eletromiográfica em músculos principais da marcha ${ }^{45}$ e aumento da fibra muscular foram também observados após o TMSPC. Um estudo recente ${ }^{45}$, o uso de TMSPC teve resultado desfavorável comparada ao treino de convencional com habilidades motoras.

Dos 21 artigos ora revisados, somente três ${ }^{28,29,39}$ apresentaram uma metodologia com ensaio clínico randomizado e/ou um estudo experimental com mais de 20 pacientes. As outras pesquisas eram relatos de caso, casocontrole, estudo piloto e/ou estudo experimental com menos de 20 pacientes. Isto demonstra, que apesar de muitos resultados positivos do TMSPC, ainda falta evidências científicas comprobatória desses efeitos.

Quadro 1

Pesquisas envolvendo a intervençâo do TMSPC em LM incompleta

\begin{tabular}{|c|c|c|}
\hline AUTORES & MÉTODO & RESULTADOS \\
\hline 1- Werning et al. $(1992)^{27}$ & $\begin{array}{l}8 \text { pacientes com LM incompleta (com } 5 \text { - } 20 \text { anos pós-lesão), } \\
\text { tempo de tratamento foi de } 11 / 2 \text { a } 7 \text { meses com sessóes de } 30 \text { - } \\
60 \mathrm{~min} \text {. ( } 5 \mathrm{x} / \mathrm{semana}) \text {. Uso do TPMSPC iniciado com } 40 \% \\
\text { do peso corporal. }\end{array}$ & $\begin{array}{l}\text { - A distância percorrida aumentou depois do treino. } \\
\text { O fenômeno importante observado foi que pacien- } \\
\text { te com atividade voluntária ausente em membro em } \\
\text { repouso conseguiu deambular por } 100 \text { a } 200 \mathrm{~m} \mathrm{(} \mathrm{au-} \\
\text { tomatismo locomotor). }\end{array}$ \\
\hline 2- Werning et al. $(1995)^{28}$ & $\begin{array}{l}89 \text { pacientes ( } 44 \text { na fase crônica e } 45 \text { na fase aguda) com LM } \\
\text { incompleta foram comparados com } 64 \text { pacientes controle. O } \\
\text { tratamento com TMSPC teve uma média de } 10.5 \text { semanas } \\
(3-20 \text { semanas), com sessáo de } 30 \mathrm{~min}(5 \mathrm{x} / \text { semana). }\end{array}$ & $\begin{array}{l}\text { - Pacientes adquiriram marcha independente, e pacien- } \\
\text { te que já deambulavam melhoraram a velocidade e o } \\
\text { desempenho da marcha. Apesar disso, houve pouca } \\
\text { melhora na atividade muscular voluntária. }\end{array}$ \\
\hline 3- Gardner et al. $(1998)^{20}$ & $\begin{array}{l}\text { Homem com } 28 \text { anos, LM em C5-C6 incompleta há } 7 \text { me- } \\
\text { ses, deambulante independente por } 46 \text { m. Realizou TMSPC } \\
\text { com } 40 \% \text { do peso corporal inicialmente, por } 6 \text { semanas ( } 3 x \\
\text { por semana) com } 16 \text { sessóes totais ( } 20 \text { min., cada) }\end{array}$ & $\begin{array}{l}\text { - Melhora do desempenho na corrida e da cadência } \\
\text { da marcha. }\end{array}$ \\
\hline 4- Werning et al. $(1998)^{29}$ & $\begin{array}{l}35 \text { pacientes com LM incompleta em fase crônica realizaram } \\
\text { o TMSPC por uma média de } 12 \text { semanas (sessão de } 30 \text { min.- } \\
5 \mathrm{x} / \text { semana) e } 41 \text { pacientes com LM incompleta na fase aguda } \\
\text { realizaram TMSPC por uma média de } 10 \text { semanas (sessão de } \\
30 \mathrm{~min} .-5 \mathrm{x} / \mathrm{semana} \text { ). }\end{array}$ & $\begin{array}{l}\text { - Melhora na funçấo passando de cadeirante para de- } \\
\text { ambulante. E deambulantes melhoraram a velocidade } \\
\text { e endurance da marcha, além de manter os ganhos } \\
\text { por um período prolongado após o treino. }\end{array}$ \\
\hline
\end{tabular}


Quadro 1

continuação.

\begin{tabular}{|c|c|c|}
\hline AUTORES & MÉTODO & RESULTADOS \\
\hline 5- Nymark et al. $(1998)^{30}$ & $\begin{array}{l}\text { Estudo Piloto com } 5 \text { pacientes com LM incompleta(fase su- } \\
\text { baguda-2meses pós-lesão) lesão entre C2 e T-10.Todos par- } \\
\text { ticipantes realizaram o TMSPC por } 36 \text { sessóes ( } 1 \text { hora cada) } \\
\text { ao longo de } 3 \text { meses. }\end{array}$ & $\begin{array}{l}\text { - Melhora na Amplitude de Movimento e nas medidas } \\
\text { cinemáticas (temporais e espaciais) da marcha. Maior } \\
\text { descarga de peso em MMII. }\end{array}$ \\
\hline $\begin{array}{l}\text { 6- Bherman, Harkema } \\
(2000)^{31}\end{array}$ & $\begin{array}{l}\text { Realizaram o TMSPC em } 3 \text { pacientes com lesão incompleta } \\
\text { ASIA C e D. } \\
\text { - Caso 1- Homem de } 20 \text { anos, lesão em T-5, ASIA C, } \\
\text { um mês após o evento, com músculos chaves pontua- } \\
\text { dos em } 2 \text { de } 50 \text { em membros inferiores. } \\
\text { Caso 2- Homem de } 43 \text { anos, lesão em C6, } 8 \text { meses } \\
\text { após o trauma, ASIA D, músculos chaves. pontuados } \\
\text { em } 32 \text { de } 50 \text {. } \\
\text { Caso 3- Homem de } 45 \text { anos, lesão T-9 ASIA D com } \\
\text { músculos chaves de MMII pontuados de 46/50. }\end{array}$ & $\begin{array}{l}\text { - Caso 1- Melhorou velocidade da caminhada e in- } \\
\text { dependência em casa, passando a usar apenas uma } \\
\text { bengala para mobilidade. -Após o treino completo, } \\
\text { progrediu para ASIA D e os músculos chaves melho- } \\
\text { raram para 38/50. } \\
\text { Caso 2- Após o treinamento, permaneceu em ASIA } \\
\text { D com melhora da pontuaçáo dos músculos chaves } \\
\text { para 34/50. -Após treino completo, se tornou deam- } \\
\text { bulante com muletas por tempo integral. Melhora na } \\
\text { velocidade da marcha, no equilíbrio, risco de queda e } \\
\text { qualidade de vida. } \\
\text { Caso 3- Náo obteve mudança na classificação e no } \\
\text { escore motor da ASIA, melhorou par subir escadas e a } \\
\text { velocidade da marcha. }\end{array}$ \\
\hline 7- Protas et al. $(2001)^{32}$ & $\begin{array}{l}\text { Estudo Piloto realizado com } 3 \text { homens com LM incompleto, } \\
\text { nível torácico. } 2 \text { casos ASIA D e } 1 \text { caso ASIA C. TMSPC foi } \\
\text { realizado com início em } 40 \% \text { do peso corporal com sessóes } \\
\text { de } 20 \text { minutos } 5 x / \text { semana durante } 3 \text { meses. }\end{array}$ & $\begin{array}{l}\text { Todos os } 3 \text { pacientes adquiriram melhora na velocida- } \\
\text { de da marcha e eficiência, diminuiçáo do consumo de } \\
\text { O2 em } 68 \% \text {.A função muscular não sofreu alteração. }\end{array}$ \\
\hline 8- Field-Fote et al $(2001)^{33}$ & $\begin{array}{l}\text { Avaliaram a TMSPC associado à eletroestimulaçáo em nervo } \\
\text { fibular. } 19 \text { pacientes com LM ASIA C com menos } 1 \text { ano pós- } \\
\text { lesáo.A duraçáo do tratamento foi de } 3 \text { meses ( } 3 x / \text { semana) } \\
\text { com sessóes de } 1.5 \text { hora. }\end{array}$ & $\begin{array}{l}\text { - Indivíduo com alguma capacidade para deambulaçáo } \\
\text { se beneficia do TMSPC associado à eletroestimuala- } \\
\text { çáo com melhora velocidade da marcha. }\end{array}$ \\
\hline 9- Field-Fote et al. $(2002)^{22}$ & $\begin{array}{l}\text { Avaliaram o efeito do TMSPC associado à Eletroesitmulaçâo } \\
\text { em nervo fibular. } 14 \text { casos e } 3 \text { controles com LM ASIA C, } \\
\text { lesão abaixo de T-10, com media de } 70 \text { meses pós-lesão. } 12 \\
\text { semanas de tratamento ( } 3 \mathrm{x} / \text { semana) com total de } 36 \text { sessôes. }\end{array}$ & $\begin{array}{l}\text { - Pacientes caminharam com uma velocidade de } 84 \% \\
\text { superior a inicial e melhoraram a coordenaçáo mo- } \\
\text { tora. }\end{array}$ \\
\hline 10- Júnior et al. $(2003)^{34}$ & $\begin{array}{l}\text { Utilizaram TMSPC em } 6 \text { pacientes com LM entre C4-T9, } \\
\text { com média de lesão de } 5.5 \text { anos. }\end{array}$ & $\begin{array}{l}\text { - Melhora na velocidade da marcha, distância percorri- } \\
\text { da e aumento no tamanho dos passos. } \\
\text { Uma sensação de aquecimento cansaço muscular na } \\
\text { regiăo de quadríceps, glúteos médios, íliopsoas e tibial } \\
\text { anterior. }\end{array}$ \\
\hline 11- Postans et al. $(2004)^{35}$ & $\begin{array}{l}12 \text { indivíduos com LM incompletas (ASIA C e D), que } \\
\text { estavam em uma fase aguda. O tempo de tratamento com } \\
\text { TMSPC associado à eletroestimulação foi de quatro semanas } \\
(5 \text { sessóes/semana) com sessóes de } 25 \text { minutos, da mesma } \\
\text { forma ocorreu no período controle realizado com fisioterapia } \\
\text { convencional. }\end{array}$ & $\begin{array}{l}\text { O grupo que recebeu TMSPC obteve melhor resulta- } \\
\text { do no desempenho e na velocidade da marcha com- } \\
\text { parada ao controle. Além disso, a espasticidade e a } \\
\text { força muscular não sofreram diferenças após ambos } \\
\text { os tratamentos. }\end{array}$ \\
\hline 12- Stewart et al. $(2004)^{36}$ & $\begin{array}{l}\text { Estudaram } 9 \text { pacientes com LM ASIA C que realizaram } 68 \\
\text { sessōes de TMSPC ( } 3 \mathrm{x} / \text { semana). Tempo da sessão não foi re- } \\
\text { latado. Iniciaram com } 65 \pm 3 \% \text { do peso corporal }\end{array}$ & $\begin{array}{l}\text { - Aumento na área de fibra muscular do tipo I e IIa. } \\
\text { Melhora do perfil lipídico (diminuiçấo do LDL).Sem } \\
\text { alteraçáo da gordura corporal. }\end{array}$ \\
\hline 13- Philps et al. $(2004)^{37}$ & $\begin{array}{l}\text { Estudaram } 9 \text { pacientes com LM, ASIA C e fizeram o trata- } \\
\text { mento com TMSPC por } 6 \text { meses ( } 3 x / \text { semana) com } 68 \text { ses- } \\
\text { sóes totais Iniciaram com } 65 \pm 3 \% \text { do peso corporal. }\end{array}$ & $\begin{array}{l}\text { - Melhorou a regulação da glicose sanguínea (aumento } \\
\text { da tolerância à glicose) e aumento da sensibilidade à } \\
\text { insulina }\end{array}$ \\
\hline 14- Behrman et al. $(2005)^{38}$ & $\begin{array}{l}\text { Analisou um paciente de } 55 \text { anos, com lesão em C6-7, ASIA } \\
\text { D, deambulava há } 3 \text { anos com um andador com rodas e uma } \\
\text { órtese AFO direita. Foram realizadas } 45 \text { sessōes de treino lo- } \\
\text { comotor, sendo } 5 \text { sessóes por semana, } 30 \text { min. de treino com } \\
\text { SPC, } 20 \text { min. de caminhada no solo e treino de deambulaçáo } \\
\text { na comunidade. }\end{array}$ & $\begin{array}{l}\text { - Evoluiu de deambulação domiciliar com auxílio de } \\
\text { órtese para deambulaçáo comunitária com uso de } \\
\text { muletas, além de melhorar a velocidade da macha, } \\
\text { tornando mais simétrica, com balanço de membros } \\
\text { superiores, coordenaçáo e passos adequados. A quan- } \\
\text { tidade de passos medidos durante } 24 \text { horas também } \\
\text { aumentou. }\end{array}$ \\
\hline 15- Field-Fote et al. $(2005)^{39}$ & $\begin{array}{l}27 \text { pacientes com LM incompleta com mais de um ano de } \\
\text { lesão, lesão abaixo de T-10 receberam o tratamento com } \\
\text { TMSP, durante } 12 \text { semanas ( } 5 \mathrm{x} / \text { semana) com sessóes de } 60 \\
\text { minutos. }\end{array}$ & $\begin{array}{l}\text { Melhora observada na velocidade da marcha, compri- } \\
\text { mento do passo e simetria. }\end{array}$ \\
\hline 16- Effing et al. $(2006)^{40}$ & $\begin{array}{l}\text { Estudo caso controle composto por } 3 \text { homens com LM crô- } \\
\text { nica ( }>4 \text { anos de lesão), } 2 \text { ASIA C e } 1 \text { ASIA D. Realizaram } \\
\text { o TMSPC por } 12 \text { semanas }(5 x / \text { semana) com sessão de } 30 \\
\text { minutos. }\end{array}$ & - Houve pequena melhora na qualidade de vida. \\
\hline
\end{tabular}


Quadro 1

continuação.

\begin{tabular}{|c|c|c|}
\hline AUTORES & MÉTODO & RESULTADOS \\
\hline 17- Lucareli et al. $(2007)^{41}$ & $\begin{array}{l}\text { Estudo prospectivo randomizado simples cego com } 2 \text { gru- } \\
\text { pos } 12 \text { pacientes cada. Todos pacientes com LM incomple- } \\
\text { ta ASIA C e D com pelo menos } 12 \text { meses pós-lesão. Foram } \\
\text { realizadas } 30 \text { sessóes ( } 2 x / \text { semana) durante } 4 \text { meses com } \\
\text { TMSPC, e no grupo controle fisioterapia convencional o } \\
\text { mesmo procedimento foi realizado. }\end{array}$ & $\begin{array}{l}\text { Aumento da velocidade distância percorrida, cadên- } \\
\text { cia, comprimento do passo, tempo total de ciclo e di- } \\
\text { minuiçấo do tempo de apoio na marcha. O TMSPC } \\
\text { foi mais efetivo que a fisioterapia convencional nos } \\
\text { parâmetros cinemáticos da marcha. }\end{array}$ \\
\hline 18- Lucareli et al. $(2008)^{42}$ & $\begin{array}{l}\text { Avaliaram o uso do TMSPC em } 12 \text { pacientes com LM ASIA } \\
\text { C e D, deambulantes, mais de } 12 \text { meses pós-lesáo.O trata- } \\
\text { mento teve duraçáo de } 4 \text { meses( } 30 \text { sessôes de } 30 \text { min., cada), } \\
\text { 2x/semana. }\end{array}$ & $\begin{array}{l}\text { Houve melhora nos parâmetros temporo-espaciais } \\
\text { da marcha, mas naao houve melhora na qualidade de } \\
\text { vida. }\end{array}$ \\
\hline 19- Behrman et al. $(2008)^{43}$ & $\begin{array}{l}\text { Realizaram TMSPC com uma criança de } 4 \text { anos e meio com } \\
\text { LM incompleta ASIA C lesão C- } 7,3 \text { meses de lesão, cadei- } \\
\text { rante dependente, pontuaçấo motora de } 4 / 50 \text { entre músculos } \\
\text { chaves. O tratamento foi de } 16 \text { meses, com total de } 76 \text { ses- } \\
\text { sóes de } 20 \text { a } 30 \text { minutos de TMSPC. }\end{array}$ & $\begin{array}{l}\text { - Logo após o } 1^{\circ} \text { mês de tratamento apresentou: passos } \\
\text { voluntários, progredindo com habilidade para deam- } \\
\text { bular com andador com rodas. No final do treino lo- } \\
\text { comotor, andava independente, com melhora signifi- } \\
\text { cativa da velocidade da marcha. Não houve mudança } \\
\text { no escore dos músculos chaves. }\end{array}$ \\
\hline 20- Musselman et al.(2009) $)^{44}$ & $\begin{array}{l}\text { Avaliaram } 4 \text { pacientes com LM ASIA C. Todos pacientes } \\
\text { iniciaram o tratamento com TMSPC. Logos depois, } 2 \text { deles } \\
\text { receberam o tratamento com treino de habilidades (andar em } \\
\text { diferentes superfícies, treino de marcha com obstáculo, an- } \\
\text { dar longas distâncias e outros) e o restante continuou com o } \\
\text { TMSPC. Na terceira fase do tratamento, inverteu-se a ordem } \\
\text { do tratamento. Cada fase do tratamento teve duraçáo de } 3 \\
\text { meses, } 1 \text { hora cada sessão ( } 5 \times \text { /semana). }\end{array}$ & $\begin{array}{l}\text { - Não houve melhora no equilíbrio estático e/ou dinâ- } \\
\text { mico. A velocidade da marcha melhorou durante o } \\
\text { tratamento com habilidades motoras, sendo superior } \\
\text { ao TMSPC. }\end{array}$ \\
\hline 21- Gorassini et al. $(2009)^{45}$ & $\begin{array}{l}9 \text { pacientes com LM ASIA C e D com mais de } 8 \text { meses pós- } \\
\text { lesáo e } 8 \text { pacientes saudáveis realizaram uma média de } 14 \\
\text { semanas de TMSPC, com sessóes de } 1 \text { hora, por } 5 \text { vezes } \\
\text { por semana. }\end{array}$ & $\begin{array}{l}\text { - Músculo tibial anterior e iquiotibial apresentaram } \\
\text { maior ativaçáo na eletromiografia. }\end{array}$ \\
\hline
\end{tabular}

Recuperar a marcha é uma tarefa difícil e dispendiosa, os pacientes muitas vezes são incapazes de produzir a força muscular necessária para manter a postura e caminhar ${ }^{4}$. Restaurar a deambulação requer variadas técnicas e geralmente exige assistência considerável do terapeuta para segurar o peso do paciente e aumentar seu equilíbrio. No treino de marcha convencional, muitas vezes, o resultado não satisfaz o paciente, com padróes assimétricos de movimento e, principalmente, com dificuldade de percorrer maiores distâncias ${ }^{6,7}$.

A base teórica para o surgimento do TMSPC advém dos estudos com gatos com lesão medular que passaram por este treino de marcha ${ }^{15}$. A partir destes estudos, foi constatado que existia no sistema nervoso dos animais um centro gerador de padrão que era responsável por gerar o padrão cíclico da marcha para estes animais com um pouco de treino mesmo após a lesão medular ${ }^{16-18}$.

Este treino tem o objetivo de gerar um padrão central e foi criado para aperfeiçoar o processo da marcha, deu-se então, início à prática de reabilitação na esteira e, mais tarde, o treino de marcha na esteira com suporte de peso corporal (TMSPC) ${ }^{8}$.

Existe uma maior facilidade para o uso do TMSPC do que o treino de marcha convencional na esteira ${ }^{11}$. Esta facilidade é gerada pelo apoio de tronco que o suporte gera $^{12}$. Com este apoio de tronco, existe maior segurança sem o risco ou temor de queda pelo paciente ${ }^{46}$. A suspensão permite uma variação na carga que os membros inferiores irão suportar, gerando a oportunidade de o paciente caminhar nas condiçóes que são necessárias para que ele possa realizar este movimento ${ }^{24,33}$.

Se por um lado a lesão medular pode desencadear importantes comprometimentos à locomoção humana, por outro, estudos recentes demonstram respostas efetivas ao treinamento em esteira rolante, com suporte de peso corporal, resultando na melhora dos padróes locomotores, tanto em animais ${ }^{47,48}$ como seres humanos com lesão medular incompleta ${ }^{49,50}$.

A medula integra-se a informação supra-espinhal aferente e com prática repetitiva pode melhorar o output 
motor. Portanto, as atividades baseadas em terapia especificamente provêem ativação do sistema neuromuscular abaixo do nível da lesão com o objetivo de "retreinar" o sistema nervoso e recuperar tarefas motoras específicas ${ }^{51}$.

Cientistas examinaram o papel da medula de controlar a caminhada e descobriram que gatos com transecção medular média torácica completa poderiam voltar a dar passos, resposta essa depois de intensa prática da tarefa de caminhar. O caminhar foi facilitado com a assistência manual com sustentação parcial do tronco, o treino envolvia aperfeiçoar uma marcha normal incluindo parâmetros de velocidade de caminhada e apropriada cinemática ${ }^{51}$.

Com o uso do TMSPC se obtém melhores resultados comparado ao treino convencional de marcha ${ }^{8,9,17,18,24}$. Em um ensaio clínico randomizado (estudo piloto) ${ }^{8}$, os autores compararam a intervenção fisioterapêutica convencional (sessóes diárias e com três horas de duração) com o TMSPC (segunda a quinta com sessôes de 20 min.), em 15 pacientes que sofreram Acidente Cerebrovascular (AVC) na fase aguda ( $<$ de 6 semanas). Como parâmetros de avaliação foram usados: capacidade funcional da marcha, a velocidade obtida em 5 metros, a distância percorrida em 5 minutos e o dispêndio de energia (consumo de oxigênio- $\mathrm{Vo}_{2}$ ). $\mathrm{O}$ tamanho da amostra não permitiu significante estatística, mas foi capaz de observar grandes diferenças na distância percorrida e no dispêndio de energia do grupo que receberam o TMSPC em relação à fisioterapia convencional.

Em outro estudo com pacientes pós-AVC, 43 pacientes receberam o tratamento com TMSPC e 36 pacientes foram tratados com treino de marcha convencional na esteira'. Os dois grupos foram atendidos por 6 semanas ( 2 sessóes de 20 min por semana). O equilíbrio, recuperação motora, velocidade e resistência da marcha foram comparadas. Houve diferenças estatísticas em todos os parâmetros, com melhores resultados no grupo que recebeu TMSPC. Conclui-se que a estratégia de ir aumentando o peso corporal ao poucos na esteira é mais vantajosa, e melhor contribui para o equilíbrio e na capacidade da marcha quando comparada a treino convencional na esteira.

Em pacientes com paralisia cerebral, o TMSPC mostrou também atuar nas habilidades da marcha e motoras, postura, equilíbrio e melhora na pontuação na escala GMFM $^{17}$ (medida da função motora grossa). É importante notar neste estudo que de 10 pacientes, 6 eram não-deambulantes, o que demonstra a eficiência do TMSPC também em pacientes dependentes na marcha.

$\mathrm{Na}$ Doença de Parkinson, o TMSPC também obteve um melhor resultado que a fisioterapia convencional. Dez pacientes com DP foram analisados e observaram uma diferença estatística entre o grupo que recebeu o TMSPC e a fisioterapia convencional, em relação a melhora na pontuação total da UPDRS, ou seja, no desempenho motor e mobilidade da marcha, além de melhorar as atividades de vida diárias ${ }^{18}$.

A TMSPC apresentou resultados negativos, não sendo benéfico para a melhora da marcha. Foram estudados 16 indivíduos com mais de 12 meses de $\mathrm{AVC}^{7}$. O tratamento durou 12 semanas (4 vezes por semana) com a realização de 90 minutos cada sessão. Um laboratório da marcha foi usado com medidas cinemáticas da marcha com uso de um computador e um programa Vicon 370, além de sistema de dados em 3D. Em um grupo (9 pacientes) foi usado TMSPC associado com estimulação elétrica, e o outro grupo (7 pacientes) somente o TMSPC. Neste último grupo, não houve ganhos estatisticamente significativos na marcha.

Vinte e sete pacientes com LM incompletas com mais de 1 ano do diagnóstico, com lesóes acima de T-10 e com capacidade para dar pelo menos um passo com uma das pernas foram avaliados em um laboratório de marcha com 8 câmeras. Após 12 semanas (5 sessóes/semana) de tratamento com TMSPC houve melhora na velocidade da marcha, no comprimento e simetria do passo ${ }^{39}$.

Outra pesquisa analisou 12 indivíduos com LM incompletas (ASIA C e D), que estavam em uma fase aguda $^{35}$. Cada paciente foi em um momento o caso e em outro momento controle. No período de controle, recebiam tratamento fisioterapêutico padrão e no controle realizavam TMSPC, com o início de $40 \%$ de retirada do peso corporal, associado com eletroestimulação. O tempo de tratamento foi de quatro semanas ( 5 sessóes/semana) com sessóes de 25 minutos para ambos os períodos de tratamento. O grupo que recebeu TMSPC obteve melhor resultado no desempenho e na velocidade da marcha comparada ao controle. Além disso, a espasticidade e a 
força muscular não sofreram diferenças após ambos os tratamentos.

Em um estudo prospectivo randomizado simples cego com 2 grupos de 12 pacientes com LM ASIA C e D, e pelo menos 12 meses de sequela, mostrou que no grupo que recebeu atendimento (30 sessóes) com TMSPC obteve aumento na velocidade da marcha, distância, cadência, comprimento do passo e tempo total do ciclo comparado ao grupo controle (fisioterapia convencional) ${ }^{41}$.

Pacientes com LM incompleta que receberam tratamento com TMSPC em um único hospital, e após revisão de todos os prontuários, chegou-se a conclusão que o uso do TMSPC é financeiramente viável quando realizados por um fisioterapeuta, 2 técnicos e um voluntário bem treinado ${ }^{52}$.

O TMSPC também mostra resultados além da mobilidade motora. Um estudo ${ }^{36}$ com paciente com LM incompletas (ASIA C) mostraram que após 6 meses de tratamento (3 sessóes /semana) houve melhora no perfil lipídico com diminuição do LDL, aumento na área da fibra muscular do tipo I e IIa, mas não mostrou alteração na massa de gordura corporal. Foi observado em pacientes com LM incompleta, melhora na regulação da glicose sanguínea (tolerância à glicose) e aumento de sensibilidade à insulina ${ }^{37}$.

O TMSPC parece não prevenir a perda da densidade óssea tanto na fase aguda ${ }^{53}$ como na fase crônica da $\mathrm{LM}^{54}$. A TPSPC mostrou também diminuição no gasto energético ${ }^{55}$ demonstrado por meio de menor consumo de oxigênio e diminuição da frequência cardíaca após o treino ${ }^{8,20,32,56-59}$. Isso acarreta em menor chance de evento traumático como parada cardíaca ou falta de ar durante o treino e um menor cansaço durante e após o treino ${ }^{12}$.

\section{CONSIDERAÇÕES FINAIS}

O treino de marcha com suporte de peso corporal é um meio seguro e confiável que surgiu para inovar a reabilitação funcional da marcha. A maioria dos artigos analisados mostram que se obtêm melhores resultados com o treino de marcha com SPC do que com o treino convencional de marcha, produzindo efeitos fisiológicos e psicológicos favoráveis aos pacientes além de apresentarem melhoras significativas das fases da marcha.

Foi notado que os indivíduos que sofreram LM incompleta e que apresenta uma capacidade neurológica para deambular, se beneficiam muito com o TMSPC. Esse benefício parece ser ainda melhor quando associado com eletroestimulação. Não foi encontrado benefícios do TMSPC para melhora da força muscular e densidade óssea, como também para diminuir a espasticidade e gordura corporal. Há necessidade de mais estudos para comprovar a real efetividade do TMSPC na LM incompleta.

\section{REFERÊNCIAS}

1.Greve JM, Are SMJ. Reabilitação da lesão da medula espinhal. In: Greve JMA, Amatezzi MM. Medicina de reabilitação aplicada à ortopedia e traumatologia. São Paulo: Roca, 1999, p.323-24.

2.Salvador LA, Tarnhovi EG. Estudo comparativo da qualidade de vida em indivíduos com trauma raquimedular praticantes e nâo-praticantes de atividades físicas, utilizando o questionário genérico SF-36. (última atualização: 05/2009; citado em: 05/2010). Disponível em: http:// www.wgate.com.br/ conteudo/ medicinaesaude/ fisioterapia/ traumato/ raquimedular/ raquimedular.htm.

3.Ekman LL. Neurociência - Fundamentos para a Reabilitação. Rio de Janeiro: Guanabara, 2000, p.191-2.

4.Finch L, Barbeau H, Arsenault B. Influence of body weight support on normal human gait: development of a gait retraining strategy. Phys Ther 1999;71:842-55.

5.Gazzani F, Fadda A, Torre M. Ward: a pneumatic system for body relief in gait rehabilitation. IEEE Trans Rehabil Eng 2000;8:506-13.

http://dx.doi.org/10.1109/86.895954

6.Coelho JL, Abrahão F, Mattioli R. Aumento do torque muscular após tratamento em esteira com suporte parcial de peso em pacientes com hemiparesia crônica. Rev Bras Fisioter 2004;8:137-43.

7.Daly JJ, Roengk KL, Butler KM, Gansen JL, Fredrickson E, Marsolais EB, et al. Response of sagittal plane gait kinematics to weight-supported treadmill training and functional neuromuscular stimulation following stroke. J Rehabil Res Dev 2004;41:807-20.

http://dx.doi.org/10.1682/JRRD.2003.08.0120

8.Cunha IT da, Lim PA, Qureshy H, Henson H, Monga T, Protas E. Gait outcomes after acute stroke rehabilitation with supported treadmill ambulation training: a randomized controlled pilot study. Arch Phys Med Rehabi 2002;83:1258-65.

http://dx.doi.org/10.1053/apmr.2002.34267

9.Visitin, M, Barbeau H, Korner-Bitenski N, Mayo N. A new approach to retrain gait in stroke patients through body weight support and treadmill stimulation. Am Heart J 1998;29:1122-8.

10.Wilson MS, Qureshy H, Protas E, Holmes A, Krouskop TA, Sherwood AM. Equipment specifications for supported treadmill ambulation training: A technical note. J Rehabil Res Dev 2001;37:415-22.

11.Hesse S, Konrad M, Uhlenrock D. Treadmill walking with partial body weight support versus floor walking in hemiparetic subjects. Arch Phys Med Rehabil 1999;80:421-7.

http://dx.doi.org/10.1016/S0003-9993(99)90279-4

12.Sullivan KJ, Knowlton BJ, Dobkin BH. Step training with body weight support: effect of treadmill speed and practice paradigms on post-stroke loco- 
motor recovery. Arch Phys Med Rehabil 2002;83:683-91.

http://dx.doi.org/10.1053/apmr.2002.32488

13.Lindquist ARR, Silva IAB, Barros RML, Mattioli R. A influência da estimulaçáo elétrica funcional associada ao treinamento em esteira com suporte parcial de peso na marcha de hemiparéticos. Rev Bras Fisioter 2005;9:109-12. 14.Dobkin BH, Apple D, Barbeau H, Basso M, Behrman A, Deforge D, et al. Methods for a randomized trial of weight-supported treadmill training versus conventional training for Walking during inpatient rehabilitation after incomplete traumatic spinal cord injury. Neurorehabil and Neural Repair 2003;17:153-66.

\section{http://dx.doi.org/10.1177/0888439003255508}

15.Threlkeld AJ, Cooper LD, Monger BP, Craven AN, Haupt HG. Temporospatial and kinematic gait alterations during treadmill walking with body weight suspension. Gait and Posture 2003;17:235-45.

http://dx.doi.org/10.1016/S0966-6362(02)00105-4

16.Duysens J, Crommert H. Van de Neural control of locomotion; Part 1: The central pattern generator from cats to humans. Gait and Posture 1998;7:131-41. http://dx.doi.org/10.1016/S0966-6362(97)00042-8

17.Schindl MR, Kern H, Hesse S. Treadmill training with partial body weight support in nonambulatory patients with cerebral palsy. Arch Phys Med Rehabil 2000;81:301-6.

\section{http://dx.doi.org/10.1016/S0003-9993(00)90075-3}

18. Miyai I, Fujimoto Y, Veda Y, Ueda Y, Yamamoto H, Nozaki S, et al. Treadmill training with body weight support: its effect on Parkinson's disease. Arch Phys Med Rehabil 2000;81:849-52.

http://dx.doi.org/10.1053/apmr.2000.4439

19.Crommert H, Van de, Mulder T, Duysens J. Neural control of locomotion: sensory control of the central pattern generator and its relation to treadmill training. Gait and Posture 1998;7:251-63.

http://dx.doi.org/10.1016/S0966-6362(98)00010-1

20.Gardner MB, Holden MK, Keikauskas JM, Ricahrd RL. Partial body weight support with treadmill locomotion to improve gait after incomplete spinal cord injury: a single-subject experimental design. Phys Ther 1998;78:361-75.

21.Cherniack EP, Caprio D, Fletcher AA, Tuckman J. A novel device for walking training in elderly patients. Physiotherapy 1999;85:144-8.

http://dx.doi.org/10.1016/S0031-9406(05)65695-7

22.Field-Fote EC, Tepavac D. Improved intralimb coordination in people with incomplete spinal cord injury following training with body weight support and electrical stimulation. Phys Ther 2002;82:707-15.

23.Visintin M, Barbeau H. The effects of body weight support on the locomotor pattern of spastic paretic patients. Can J Neurol Sci 1989;16:315-25.

24.Barbeau H, Visintin M. Optimal outcomes obtained with body-weight support combined with treadmill training in stroke subjects. Arch Phys Med Rehabil 2003,84:1458-65.

http://dx.doi.org/10.1016/S0003-9993(03)00361-7

25.Haupenthal A, Schutz GR, Souza PV, Roesler H. Análise do suporte de peso corporal para o treino de marcha. Fisioterapia em movimento 2008;21:85-92. 26.Maynard FM Jr, Bracken MB, Creasey G, Ditunno JF Jr, Donovan WH, Ducker TB, et al. International Standards for Neurological and Functional Classification of Spinal Cord Injury. American Spinal Injury Association. Spinal Cord 1997;35:266-74.

\section{http://dx.doi.org/10.1038/sj.sc.3100432}

27.Wernig A, Müller S. Laufband locomotion with body weight support improved walking in persons with severe spinal cord injuries. Paraplegia 1992;30:229-38.

http://dx.doi.org/10.1038/sc.1992.61

28.Wernig A, Müller S, Nanassy A, Cagol E. Laufband therapy based on 'rules of spinal locomotion' is effective in spinal cord injured persons. Eur J Neurosci
1995;7:823-9.

http://dx.doi.org/10.1111/j.1460-9568.1995.tb00686.x

29. Wernig A, Nanassy A, Müller S. Maintenance of locomotor abilities following Laufband (treadmill) therapy in para- and tetraplegic persons: follow-up studies. Spinal Cord 1998;36:744-9.

http://dx.doi.org/10.1038/s.sc. 3100670

30.Nymark J, DeForge D, Barbeau H, Badour M, Bercovitch S, Tomas J, et al. Body Weight Support Treadmill Gait Training in the Subacute Recovery Phase of Incomplete Spinal Cord Injury. Neurorehabil Neural Repair 1998;12:11936.

\section{http://dx.doi.org/10.1177/154596839801200307}

31.Behrman AL, Harkema SJ. Locomotor training after human spinal cord injury: a series of studies. Phys Ther 2000;80:688-700.

32.Protas EJ, Holmes A, Qureshy H, Sherwood AM. Supported treadmill ambulation training after spinal cord injury: a pilot study. Arch Phys Med Rehábil 2001;82:825-31.

http://dx.doi.org/10.1053/apmr.2001.23198

33.Field-Fote EC. Combined use of body weight support, functional electric stimulation, and treadmill training to improve walking ability in individuals with chronic incomplete spinal cord injury. Arch Phys Med Rehabil 2001;82:818-24.

http://dx.doi.org/10.1053/apmr.2001.23752

34.Júnior CSA, Abreu CC, Biase ME, Ribeiro LHS. Treino locomotor com suporte em solo. Revista de Medicina Física e Reabilitação.(última atualização: 07/2003; citado em: 06/2010). Dispónivel em: http://www.spmfr.org.br.

35.Postans NJ, Hasler JP, Granat MH, Maxwell DJ. Functional electric stimulation to augment partial weight-bearing supported treadmill training for patients with acute incomplete spinal cord injury: a pilot study. Arch Phys Med Rehabil 2004;85:604-10.

http://dx.doi.org/10.1016/j.apmr.2003.08.083

36.Stewart BG, Tarnopolsky MA, Hicks AL, McCartney N, Mahoney DJ, Staron RS, et al. Treadmill training induced adaptations in muscle phenotype in persons with incomplete spinal cord injury. Muscle Nerve 2004;30:61-8. http://dx.doi.org/10.1002/mus.20048

37.Phillips SM, Stewart BG, Mahoney DJ, Hicks AL, McCartney N, Tang JE, et al. Body-weight-support treadmill training improves blood glucose regulation in persons with incomplete spinal cord injury. J Appl Physiol 2004;97: 716-24.

http://dx.doi.org/10.1152/japplphysiol.00167.2004

38.Behrman AL, Lawless-Dixon AR, Daviis SB, Bowdwn MG, Nair CP, Hannold EM, et al. Locomotor Training Progression and outcomes after incomplete spinal cord injury. Phys Ther 2005;85:1356-71.

39.Field-Fote EC, Lindley SD, Sherman AL. Locomotor training approaches for individuals with spinal cord injury: A preliminary report of walking-related outcomes. J Neurol Phys Ther 2005;29:127-38.

40.Effing TW, van Meeteren NL, van Asbeck FW, Prevo AJ. Body weightsupported treadmill training in chronic incomplete spinal cord injury: a pilot study evaluating functional health status and quality of life. Spinal Cord. 2006;44:287-96.

http://dx.doi.org/10.1038/sj.sc.3101841

41.Lucareli PR, Silva MM, Bernarddi AA, Greeve JMA. Esteira com suporte de peso corpóreo em pacientes com lesão medular. In: XII Congresso Brasileiro de Biomecânica, São Pedro. Anais do XII Congresso Brasileiro de Biomecânica, 2007, v.1. p. xx-xx.

42.Lucareli PR, Lima MO, Lima FP, Garbelotti SA Jr, Gimenes RO, Almeida JG, et al. Gait analysis and quality of life evaluation after gait training in patients with spinal cord injury. Rev Neurol 2008;46:406-10.

43.Behrman AL, Nair PM, Bowden MG, Dauser RC, Herget BR, Martin 
JB, et al. Locomotor Training Restores Walking in a Nonambulatory Child Whith Chronic, Severe, Incomplete Cervical Spinal Cord Injury. Phys Ther 2008;88:580-90.

http://dx.doi.org/10.2522/ptj.20070315

44.Musselman KE, Fouad K, Misiaszek JE, Yang JF. Training of walking skills overground and on the treadmill: case series on individuals with incomplete spinal cord injury. Phys Ther. 2009;89:601-11.

http://dx.doi.org/10.2522/ptj.20080257

45.Gorassini MA, Norton JA, Nevett-Duchcherer J, Roy FD, Yang JF. Changes in locomotor muscle activity after treadmill training in subjects with incomplete spinal cord injury. J Neurophysiol 2009;969-79.

46.Leães R, Dalmarco G, Russomano T, Azevedo DG, Pinho M, Gurgel J, et al. Avaliação da marcha humana em terra e em simulaçôes de reduçấo da força gravitacional. In: Anais do $11^{\circ}$ Congresso Brasileiro de Biomecânica, João Pessoa, 2005, p.1-5.

47.Edgerton VR, De Guzman CP, Gregor RJ, Roy RR, Hodgson JA, Lovely RG. Trainability of the spinal cord to generate stepping patterns in adult spinalized cats. In: Shimamura M, Grllner S, Edgerton V.R. editors.; Neurobiological Basis of Human Locomotion. Japan Scientific Society Press, 1991, p.411-23.

48.De Guzman CP, Hodgson JRR, Edgerton VR. Coordination of motor pools controlling the ankle musculature in adult spinal cats during treadmill walking. Brain Res 1991;555:202-14.

http://dx.doi.org/10.1016/0006-8993(91)90343-T

49.Dietz V, Muller R, Colombo G. Locomotor activity in spinal man: significance of afferent input from joint and load receptors. Brain 2002;125:262-348. http://dx.doi.org/10.1093/brain/awf273

50.Monteiro W, Fregni FSM, Fatarelli, IFC. Evolução Clínica da Marcha em um indivíduo com Lesão Medular Incompleta: Relato de Caso. Revista Fisioterapia e Pesquisa da Universidade de São Paulo 2006;13:17-23.
51.Behrman AL, Harkema SJ. Physical Rehabilitation as an Agent for Recovery After Spinal Cord Injury. Phys Med Rehábil Clin N Am 2007;18:183-202. http://dx.doi.org/10.1016/j.pmr.2007.02.002

52.Morrison SA, Backus D. Locomotor Training: Is Translating Evidence Into Practice Financially Feasible? JNPT 2007;31:50-5.

53. Giangregorio LM, Hicks AL, Webber CE, Phillips SM, Craven BC, Bugaresti JM, et al. Body weight supported treadmill training in acute spinal cord injury:Impact on muscle and bone. Spinal Cord 2005;43:649-57.

http://dx.doi.org/10.1038/sj.sc.3101774

54.Giangregorio LM, Webber CE, Phillips SM, Hicks AL, Craven BC, Bugaresti JM, et al. Can body weight supported treadmill training increase bone mass and reverse muscle atrophy in individuals with chronic incomplete spinal cord injury? Appl Physiol Nutr Metab. 2006;31:283-91.

http://dx.doi.org/10.1139/h05-036

55.Griffin TM, Tolani NA, Kram R. Walking in simulated reduced gravity: mechanical energy fluctuations and exchange. J Appl Physiol 1999;86:383-90. 56. Hunter D, Cole E, Murray JM. Energy expenditure of below-knee amputees during harnesssupported treadmill ambulation. J Orthop Sports Phys Ther 1995;21:268-76.

57.Mangione KK, Axen K, Haas F. Mechanical unweighting effects on treadmill exercise and pain in elderly people with osteoarthritis of the knee. Phys Ther 1996;76:387-95.

58.Danielson A, Sunnerhagen KS. Oxygen consuption during treadmill walking with and without body weight support in patients with hemiparesis after stroke and in healthy subjects. Arch Phys Med Rehabil 2000;81:953-7. http://dx.doi.org/10.1053/apmr.2000.6283

59.Hesse S, Werner C, Paul T, Bardeleben A, Chaler J. Influence of walking speed on lower limb muscle activity and energy consumption during treadmill walking of hemiparetic patients. Arch Phys Med Rehabil 2001;82:1547-50. http://dx.doi.org/10.1053/apmr.2001.26607 